\title{
Signalisation apototique induite par les LDL oxydées Implication dans I'athérosclérose
}

Anne NEGRE-SALVAYRE

Cécile VINDIS

Robert SALVAYRE

Inserm U-466 et

Laboratoire de biochimie médicale,

Institut Louis Bugnard,

Faculté de Médecine,

Université Paul Sabatier,

Toulouse, France.

Fax : +33-561-32-20-84

<anesalv@toulouse.inserm.fr>

\begin{abstract}
The balance between vascular cell proliferation vs apoptosis plays a key role in vessel wall remodeling. Intimal migration and proliferation of smooth muscle cells (SMC), and secretion of extracellular matrix are involved in fibrous cap formation and plaque stability, whereas apoptosis of vascular cells may contribute to the erosion and instability of the plaque leading to its rupture and subsequent thrombus formation. LDL become atherogenic after undergoing oxidation within the vascular wall. Oxidized LDL (oxLDL) and oxidized lipids exhibit complex biological properties involved in endothelial dysfunction, SMC migration and proliferation, inflammation, and apoptosis. Oxidized LDL-induced apoptosis involves the extrinsic propapoptotic pathway (linked to Fas/Fas ligand) in lymphocytes, and the intrinsic mitochondrial apoptotic pathway, involving bcl-2 family members, cytochrome $\mathrm{C}$ release, and the terminal executive caspase-3 pathway, as well as the mitochondrial apoptotic factor AIF, in vascular cells. The apoptotic signaling of ox LDL is mediated in part by an intense and sustained rise of cytosolic calcium. The mechanisms regulating the balance between proliferation and apoptosis triggered by oxLDL and their role in vivo in atherosclerotic plaque progression remains to be clarified.
\end{abstract}

Key words: oxidized LDL, apoptosis, necrosis, atherosclerosis, cell signaling, calcium

LDL oxydées induisent la prolifération des $\mathrm{CML}$, via diverses voies de signalisation [13], telles que la voie sphingomyéline/céramide/ sphingosine-1-phosphate (Spm/Cer/S1P), impliquée dans la synthèse d'ADN, et la voie EGFR/PI3K/Akt, impliquée dans la survie [14, 15], ainsi que la génération de radicaux oxygénés intracellulaires (ROS) qui participent à l'activation du récepteur au PDGF et à l'activation de NFKB par les LDL oxydées $[16,17]$.

À forte concentration, les LDL oxydées sont cytotoxiques pour les cellules vasculaires et induisent de la nécrose ou de l'apoptose, en fonction de leur concentration, du type cellulaire et l'expression de Bcl2 [18]. La nécrose primaire est caractérisée par un gonflement et des altérations des organelles intracellulaires et la libération des composants cytosoliques dans le milieu extracellulaire $[19,20]$. L'apoptose implique une condensation du cytoplasme et du noyau, une fragmentation de l'ADN et de la cellule sans altération de la membrane plasmique [19]. Plusieurs mécanismes sont impliqués dans l'apoptose, parmi lesquels le système extrinsèque impliquant les récepteurs à domaine de mort (death domain receptors) tels que Fas/Fas ligand, ou le TNF récepteur, suivi de la formation du DISC (death-inducible signaling complex) qui active caspase-8 et la cascade de caspases conduisant à caspase-3 [19-22]. L'autre système d'apoptose dit intrinsèque, implique la voie mitochondriale, les membres proapoptotiques de la famille $B C 12$, la libération de cytochrome $C$ et l'activation de caspase-3 [23]. Les LDL oxydées activent les voies extrin- sèques et mitochondriales, ainsi que des voies conduisant à la nécrose [4, 21-23].

Cette revue fait le point sur les mécanismes impliqués dans l'apoptose induite par les LDL oxydées et leur relevance dans la stabilité/ instabilité de la plaque.

\section{Composés bioactifs impliqués dans la signalisation apoptotique des LDL oxydées}

L'oxydation des LDL est un évènement majeur dans la paroi vasculaire, car il est à la base de leur athérogénicité. Les LDL sont transformées par l'oxydation en véritables cytotoxines capables d'induire des signalisations inflammatoires, mitogènes ou toxiques, et de promouvoir des évènements athérogènes majeurs [24]. Les LDL oxydées sont présentes dans les lésions d'athérosclérose [25]. L'oxydation des LDL peut être obtenue in vitro par contact avec les cellules vasculaires en culture qui génèrent des radicaux libres oxygénés en présence de métaux de transition [25-27]. L'oxydation des LDL est un processus progressif qui conduit à la formation, dans un premier temps, de LDL oxydées a minima, très bioréactives et oxydées essentiellement sur la partie lipidique [26-28]. L'oxydation des LDL induit la formation de produits d'oxydation lipidiques tels que des aldéhydes (malondialdehyde ou MDA, 4-hydroxynonenal ou 4-HNE, acroléine), des oxystérols et des hydroperoxy-cholesterol esters, des phospholipides oxydés, des hydroperoxy-phospholipides, des sphingolipi- 
des et des lysophospholipides) [27]. Les oxystérols, en particulier le 7-kétocholesterol, mais également les $25-\mathrm{OH}$ et $7-\mathrm{OH}$ cholestérols (les plus abondants parmi les oxystérols présents dans les LDL oxydées) sont responsables pour une large part des effets biologiques des LDL (inflammation, immunosuppression, régulation du métabolisme du cholestérol, cytotoxicité), et sont proapoptotiques [29-31].

Les acides gras et les phospholipides oxydés (13-HPODE) ont des effets paradoxaux, pro- ou anti-inflammatoires [32-34]. Le 4-HNE et l'acroléine ne sont pas ou peu impliqués dans la réponse inflammatoire, mais sont prolifératifs ou proapoptotiques, en fonction de leur concentration in situ [27, 35]. Les lysophospholipides et les sphingolipides (céramide, sphingosine, sphingosine 1-phosphate) sont formés dans les LDL oxydées ou les cellules, et participent aux réponses cellulaires (prolifération ou apoptose) [36-39].

En fait, il serait très important d'évaluer la part relative de chaque composant dans les propriétés générales des LDL oxydées, car leur taux respectif peut varier en fonction du mécanisme d'oxydation, du temps, et de façon interindividuelle. Le rôle respectif de chacun des lipides présents dans les $L D L$ oxydées, reste à démontrer.

\section{Mécanismes de toxicité induits par les LDL oxydées}

\section{Captation des LDL oxydées}

Les LDL sont le principal transporteur de cholestérol du sang vers les tissus, via le récepteur apoB/E [40]. L'oxydation des LDL est un processus progressif qui conduit d'abord à la formation de LDL oxydées a minima. Ces LDL sont très bioréactives, capables d'induire diverses voies de signalisation et de réponses cellulaires, et se comportent comme de véritables cytotoxines ou « Cheval de Troie » car elles peuvent introduire dans la cellule des produits d'oxydation lipidique sans altération notable de leur captation par le récepteur apoB/E [24].

À côté du récepteur apoB/E, les cellules macrophagiques et vasculaires expriment des récepteurs scavengers tels que LOX-1 ou CD36 [41-43]. Les récepteurs scavengers de type A (SR-Al and SR-All) captent les LDL plus fortement oxydées [43] et participent à la signalisation athérogène des LDL oxydées.

\section{Rôle des cavéoles et de la cavéoline}

Les cavéoles sont des invaginations de la membrane plasmique, riches en cholestérol et en sphingolipides associés aux cavéolines, protéines caractéristiques des cavéoles. Les cavéoles jouent un rôle important dans la modulation de la signalisation athérogène, du fait de la locali- sation intracavéolaire de nombreuses molécules de signalisation. Les cavéoles et la cavéoline sont présents dans les macrophages et les $S M C$, et pourraient jouer un rôle pro- ou antiathérogène, en fonction du type cellulaire [44]. Leur implication dans la signalisation induite par les LDL oxydées est encore mal connue, mais semble probable du fait de la présence dans les cavéoles des récepteurs scavengers (SR-B1, CD36). De plus, l'expression de caveoline-1 dans les cellules endothéliales, pourrait potentialiser leur sensibilité à l'apoptose induite par les LDL oxydées.

\section{Stress oxydant}

Les espèces réactives de l'oxygène (ROS), sont générées par un très grand nombre $d^{\prime}$ agents et induisent diverses réponses cellulaires telles que la prolifération, la survie ou l'apoptose, via des voies de signalisation telles que les MAPK (ERK1/2, SAPK, JNK), NF-kB, Akt, les caspases, ou le calcium [45]. Les LDL oxydées induisent la génération de ROS dans divers types cellulaires [46-48], peut-être via le récepteur LOX-1 [49]. Les sources impliquées dans la génération de ROS ne sont pas élucidées mais pourraient impliquer les peroxisomes, la $\mathrm{NAD}(\mathrm{P}) \mathrm{H}$ oxydase, les mitochondries ou les lipoxygénases [48-50]. Les LDL oxydées induisent également une diminution d'expression des enzymes antioxydantes telles que la $\mathrm{Cu} / \mathrm{Zn}$ superoxide dismutase et la glutathion peroxydase [51-53], ce qui contribue à maintenir un niveau de ROS intracellulaire élevé. Les ROS participent largement à la signalisation induite par les LDL oxydées, en particulier dans la prolifération (activation de récepteurs à tyrosine kinase et de facteurs de transcription tels que NF-kB) $[15-17,54]$. Le rôle des ROS dans la toxicité des LDL est basé sur l'observation que les antioxydants ou la surexpression d'enzymes antioxydantes protègent de la mort induite par les LDL [52-56]. Cependant, le rôle précis des ROS et de leurs cibles reste à élucider.

\section{Voie sphingomyeline/ceramide}

Les voies de signalisation associées aux cycles des sphingolipides sont complexes et encore mal connues, car la nature des enzymes impliquées (sphingomyelinases) n'est pas élucidée, de même que le(s) mécanisme(s) d'activation de cette voie [57]. L'activation de la sphingomyelinase neutre (smpd3) par les LDL oxydées, suivie de l'activation de sphingosine kinase, est impliquée dans l'effet mitogène des LDL, et implique en amont l'activation des métalloprotéases MMP2 et MT1-MMP [58, 59]. De plus, les souris fro, qui présentent une mutation sur I'activité enzymatique de smpd3 [60], de même que les souris KO pour smpd3 [61], n'ont pas de déficit en apoptose, mais présentent un retard de croissance. À l'opposé,
I'implication de sphingomyelinase acide dans l'apoptose induite par les LDL oxydées a été rapportée par plusieurs groupes [62, 63], cependant le céramide ne semble pas impliqué dans l'apoptose induite par les LDL oxydées dans les cellules endothéliales [64].

\section{Apoptose induite par les LDL oxydées}

L'effet toxique des LDL oxydées sur les cellules vasculaires se traduit par de l'apoptose ou de la nécrose en fonction des types cellulaires et de la concentration en produits d'oxydation lipidique toxiques. De plus, la toxicité est modulée par l'activation de voies de survie et l'expression de protéines pro- ou antiapoptotiques [65].

La nécrose et l'apoptose ont des caractéristiques morphologiques précises. La nécrose est caractérisée par un gonflement cellulaire, une altération des organelles, une rupture de la membrane plasmique et le relargage des composants cellulaires dans le milieu. L'apoptose se caractérise par une fragmentation du noyau et de I'ADN, une relocalisation des organelles et la formation de corps apoptotiques sans modification de perméabilité membranaire. L'apoptose implique des voies de signalisation telles que les caspases, les lysosomes ou la dérégulation du calcium $[19,65]$ figure 1 .

\section{Modification des protéines par les produits d'oxydation lipidique}

Les produits d'oxydation lipidique (MDA, acrolein, 4-HNE) peuvent réagir avec les thiols (glutathion ou autre groupements thiol), et les groupements aminés libres [27, 35]. La formation d'aldéhyde-adduits sur les protéines entraîne des dysfonctions enzymatiques, métaboliques, et de signalisation, d'où une dysfonction cellulaire pouvant induire de l'apoptose [66]. Les LDL oxydées altèrent l'activité du système ubiquitine/proteasome, ce qui entraîne une accumulation de protéines modifiées et potentialise l'effet toxique des $L D L$, et induit une inhibition de NF-kB $[67,68]$.

\section{Lysosomes}

Les LDL oxydées peuvent être dégradées dans le compartiment endolysosomal, et les lipides oxydés (oxystérols, peroxydes lipidiques...) sont relargués dans la cellule ou ils interagissent avec des cibles spécifiques. L'hydrolyse lysosomiale des LDL oxydées est plus lente que celle des LDL normales, et les lipides oxydés peuvent s'accumuler dans les lysosomes et inhiber leur fonction, puis induire leur rupture et la relocalisation des enzymes lysosomales dans le cytosol et la mort cellulaire par autophagie (endopepsis) [69-72]. 


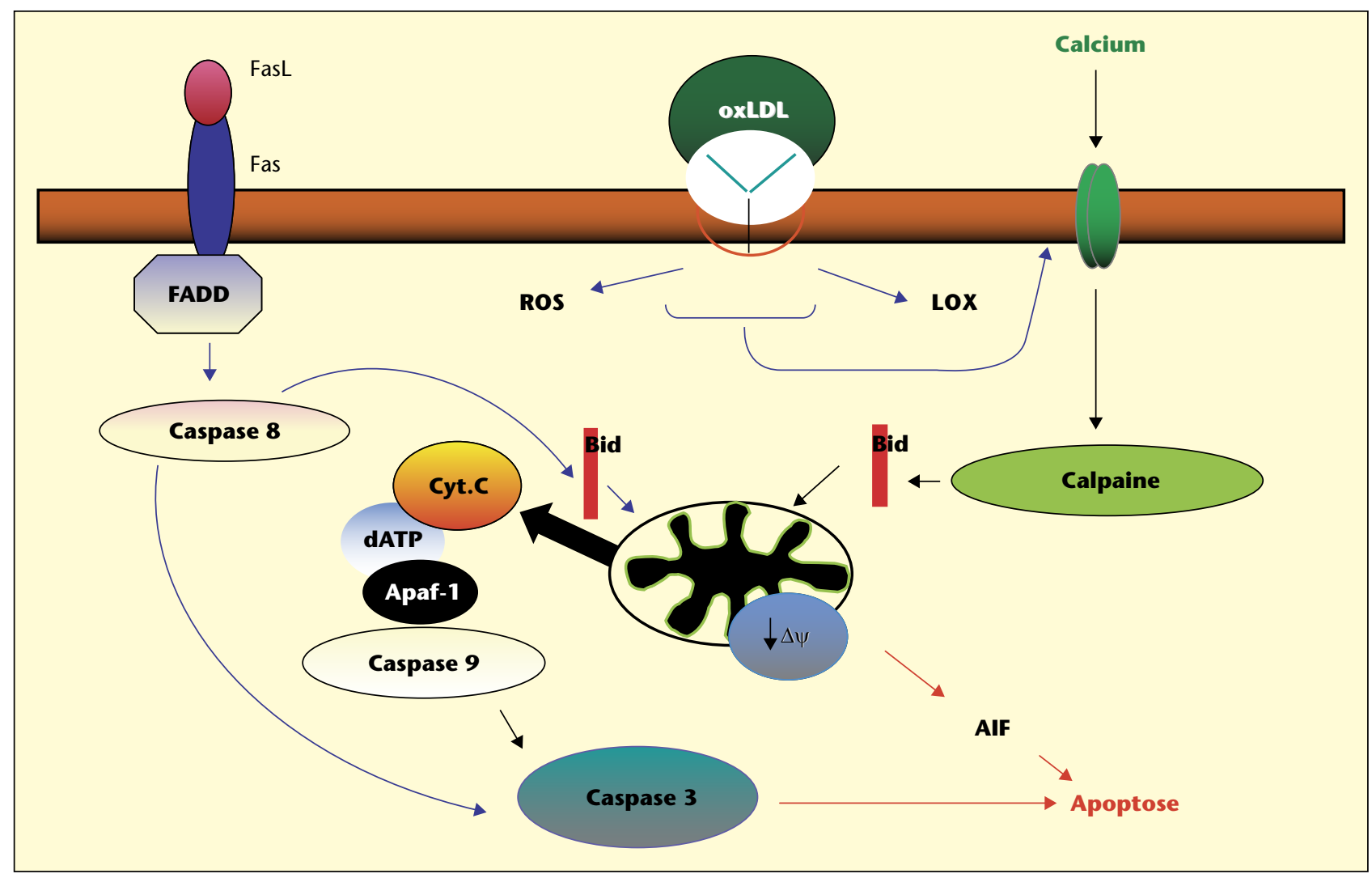

Figure 1. Effets pro-apoptotiques des LDL oxydées. ROS, espèces réactives de I'oxygène; LOX, lipides oxydés.

\section{Activation des caspases}

Les caspases jouent un rôle majeur dans I'apoptose (initiateur et effecteur), et sont impliquées dans les voies extrinsèques et intrinsèques en fonction de l'origine du stimulus [73]. La voie intrinsèque mitochondriale résulte de l'ouverture du pore de transition mitochondrial (PTP), ce qui induit un relargage de protéines proapoptotiques dans le cytosol, telles que le cytochrome $\mathrm{C}$ qui se combine avec APAF-1 (apoptosis activating factor) et pro-caspase-9, pour former l'apoptosome qui active caspase-9 puis caspase-3 [73-75]. Les membres de la famille $\mathrm{Bcl}-2$ (Bax, Bad, t-Bid) sont impliqués dans I'ouverture du PTP, alors que la cyclosporine A bloque ce mécanisme [76].

La voie extrinsèque résulte de la fixation de ligands proapoptotiques sur des récepteurs spécifiques (Fas/FasL), ce qui entraîne un recrutement de facteurs cytosoliques tels que FADD, qui form le DISC (death-inducing signaling complex). DISC active caspase-8 qui clive et active caspase-3, soit directement, soit via des crosstalks avec la voie intrinsèque mitochondriale, après clivage de Bid par caspase 8 [77].

Les LDL oxydées et les oxystérols induisent l'activation des voies intrinsèques et extrinsèques $[4,77,78]$. D'une part, les LDL oxydées stimulent l'expression du système Fas/FasL et du TNF récepteur dans les T lymphocytes [4,
$22,77,78]$. D'autre part, les LDL oxydées et les oxystérols activent la voie mitochondriale avec un relargage de cytochrome $C$ (inhibé par la cyclosporine A), et l'activation de caspase $3[4$, 79]. Les inhibiteurs de caspase (Z-VAD. fmk, Z-DVED. fmk) réduisent partiellement l'apoptose induite par les LDL oxydées [79-81]. L'apoptose induite par les LDL oxydées est inhibée par l'expression de Bcl2, mais les cellules meurent alors par nécrose [18].

\section{Calcium}

L'altération de I'homéostasie calcique est souvent observée dans la mort cellulaire et peut résulter d'un épuisement des réserves calciques intracellulaires d'où influx de calcium extracellulaire, ainsi que d'un dysfonctionnement de la fonction mitochondriale induisant une inhibition des systèmes de transport du calcium à la membrane plasmique, au niveau du réticulum endoplasmique ou de la mitochondrie [19].

\section{Calcium, ER stress et LDL oxydées}

Les perturbations métaboliques (diabète, stress oxydant, dysfonction du transport calcique) induisent des changements de conformation des protéines dans le réticulum endoplasmique (ER) [82]. L'accumulation de protéines mal conformées induit I'ER stress, ce qui permet une élimination de ces protéines. L'ER stress induit I'UPR (unfolded protein response), caractérisée par l'induction de protéines chaperonnes et de facteurs proapoptotiques tels que CHOP/GADD153 [82-84].

L'activation de l'ER stress par les LDL oxydées $n^{\prime}$ est pas connue. Une hypothèse est que l'ER stress pourrait jouer un rôle dans la dérégulation du calcium induit par les LDL oxydées et impliquée dans la mort cellulaire.

\section{Calcium, mitochondries et LDL oxydées}

Le calcium est impliqué à divers niveaux dans la signalisation mitochondriale de l'apoptose. La dérégulation du calcium stimule la phosphatase calcium/calmoduline-dépendente ou calcineurin qui déphosphoryle Bad (membre proapoptotique de la famille de $\mathrm{BCl} 2$ ), ce qui induit I'ouverture du PTP [85]. Les enzymes calcium-dépendantes (endonucléases, phospholipases, protéases, transglutaminases, calpaïnes), sont activées en conséquence du pic calcique et induisent des dommages intracellulaires irréversibles, d'où apoptose ou nécrose [19]. Les calpaïnes peuvent cliver Bid qui induit également I'ouverture du PTP $[86,87]$. Les mitochondries sont également impliquées dans le relargage de l'AlF (apoptosis inducing factor), qui transloque dans le noyau et induit un clivage de l'ADN en gros fragments [81]. 
Les LDL oxydées et les produits d'oxydation lipidique (oxystérols, aldéhydes) induisent une dérégulation du calcium cytosolique qui joue un rôle majeur dans la toxicité [86-89]. La dérégulation calcique semble due à un influx de calcium extracellulaire du fait de l'effet protecteur de l'EGTA (qui chélate le calcium extracellulaire), et des bloqueurs de canaux calciques, qui bloquent le calcium et la toxicité [86]. Le mécanisme précis impliqué dans le pic de calcium n'est pas connu, mais pourrait faire intervenir divers paramètres tels que le stress oxydant, les cavéoles/rafts, l'ER stress ou une dérégulation des pompes $\mathrm{Ca}(2+) /$ ATPases dépendantes. Le calcium est impliqué dans I'apoptose induite par les LDL oxydées via au moins deux mécanismes impliquant la voie intrinsèque mitochondriale : 1) l'activation des calpaïnes induit la dégradation de l'agent proapoptotique Bid, ce qui induit l'ouverture du PTP, le relargage de cytochrome $C$ dans le cytosol et l'activation de caspase 3 [81, 90]; 2) le relargage d'AIF dans le cytosol et sa translocation dans le noyau. L'EGTA bloque toute la signalisation apoptotique induite par les LDL oxydées, alors que les inhibiteurs de caspases ou de calpaïnes ne sont que partiellement efficaces [81].

\section{Effet des LDL oxydées sur I'expression de gènes impliqués dans l'apoptose}

Les LDL oxydées induisent l'expression d'un large panel de gènes impliqués dans la régulation de la prolifération ou l'apoptose [91]. Les LDL oxydées induisent l'expression de Fas (CD95/APO-1)/Fas ligand, qui est impliqué dans l'apoptose des lymphocytes et des cellules endothéliales [4]. L'activation de Fas induit le recrutement et l'activation de FADD (fasassociated death domain protein) et de FLICE (FADD-homologous ICE-like protease), ce qui induit une activation de la cascade de caspases [90]. La downregulation de FLIP (pour FLICE inhibitory protein) par les LDL-oxydées, augmente l'activité apoptotique du système Fas/FasL [91]. Les LDL oxydées modulent I'expression de I'IL-1 et du TNF [91-93], qui peuvent participer à l'apoptose. De plus, les LDL oxydées stimulent la surexpression de protéines proapoptotiques telles que Bax et p53 [94], et réduisent l'expression de $\mathrm{BCl}-2$ et de BCl-XL [95].

Les LDL oxydées et les lipides modulent l'activation ou l'inhibition de facteurs de transcription tels que ATF-2, ELK-1, CREB, AP-1, STAT1, STAT3 or NFKB [96-98]. Les LDL oxydées et les lysoPC ont un effet biphasique sur l'activation de NFkB qui dépend du temps de contact et de la concentration en LDL [66]. L'effet inhibiteur des LDL oxydées pourrait résulter d'une inhibition de la dégradation de IкB, (l'inhibiteur cytosolique de NFkB) par le protéasome $[93,99]$. $N F \kappa B$ régule l'expression de divers gènes impliqués dans la survie, tels que A20, cMyb, cFLIP, $\mathrm{BfI}-1 / \mathrm{A} 1, \mathrm{c}-\mathrm{IAP} 2$, xIAP, TRAF1, and BCl-xL [66]. Dans les cellules vasculaires, les LDL oxydées induisent I'inhibition de gènes antiapoptotiques, donc augmentent la sensibilité des cellules à l'apoptose [99].

\section{Conclusion}

En conclusion, la balance entre survie et mort cellulaire dans la plaque est régulée par des voies de signalisation de survie ou proapoptotiques, et les interactions entre cellules et composants de la matrice extracellulaire. Les LDL oxydées et les lipides induisent une signalisation complexe conduisant à la survie ou à la mort par nécrose ou par apoptose, en fonction de leur concentration locale, et de leur contenu (ou nature) en produits d'oxydation lipidique, de même que l'expression de gènes pro- ou antiapoptotiques, ou le type cellulaire. La signalisation propaoptotique induite par les LDL oxydées implique à la fois la voie extrinsèque activée par les récepteurs et ligands de mort cellulaires (via la surexpression de Fas/Fas ligand et du TNF récepteur), ainsi que la voie intrinsèque mitochondriale et l'activation de caspases. Le calcium joue un rôle central dans l'activation de la voie mitochondriale, et les chélateurs de calcium (EGTA) ont un effet protecteur contre la toxicité des LDL oxydées. L'effet apoptotique du calcium est médié par d'une part par les calpaïnes qui clivent et activent Bid, et donc induisent I'ouverture du PTP, le relargage de cytochrome $C$ et l'activation de caspase-3, et d'autre part par AIF. Le calcium est impliqué dans l'apoptose et la nécrose, et la surexpression de Bcl2 protège de l'apoptose mais induit un shift vers la nécrose. Les études futures devraient permettre de mieux définir la part respective de chaque produit d'oxydation dans la bioactivité totale des LDL oxydées, et d'explorer les relations entre les différentes voies de signalisation induites par les LDL oxydées et impliquées dans l'apoptose, les interconnexions entre membrane, cytosquelette et matrice extracellulaire amenant à la dérégulation du calcium, en particulier le stress du réticulum endoplasmique (RE), les dysfonctions des canaux calciques et les flux calciques RE-mitochondrie dans la signalisation apoptotique induite par les LDL oxydées [100]

Remerciements. Ce travail a reçu le soutien financier de I'Inserm et de l'université Paul Sabatier-Toulouse III.

\section{RÉFÉRENCES}

1. LUSIS. Atherosclerosis. Nature $2000 ; 407$ : 233.

2. HOLVOET P. Oxidized LDL and coronary heart disease. Acta Cardiol 2004 ; 59 : 479.

3. AIKAWAM, LIBBY P. Atherosclerotic plaque inflammation : the final frontier? Can / Cardiol $2004 ; 20: 631$.

4. NAPOLI C, QUEHENBERGER O, DE NIGRIS F, ABETE P, GLASS CK, PALINSKI W. FASEB $2000 ; 14: 1996$.

5. LITTLEWOOD TD, BENNETT MR. Apoptotic cell death in atherosclerosis. Curr Opin Lipidol $2003 ; 14: 469$.

6. CHOY JC, GRANVILLE DI, HUNT DW, MCMANUS BM. Endothelial cell apoptosis : biochemical characteristics and potential implications for atherosclerosis. Mol Cell Cardiol 2001 ; 33: 1673.

7. WILLERSON JT, RIDKER PM. Inflammation as a cardiovascular risk factor. Circulation 2004 ; 109(21 Suppl 1) : 112

8. LESKINEN M], KOVANEN PT, LINDSTEDT KA. Regulation of smooth muscle cell growth, function and death in vitro by activated mast cells a potential mechanism for the weakening and rupture of atherosclerotic plaques. Biochem Pharmacol 2003; $66: 1493$.

9. STEINBERG D, PARTHASARATHY S, CAREW TE, KHOO JC, WITZTUM JL. Beyond cholesterol. Modifications of low-density lipoprotein that increase its atherogenicity. N Engl J Med 1989 ; $320: 915$.

10. STEINBRECHER UP, ZHANG H, LOUGHEED M. Role of oxidatively modified LDL in atherosclerosis. Free Rad Biol Med $1990 ; 9$ : 155

11. BERLINER JA, HEINECKE JW. The role of oxidized lipoproteins in atherogenesis. Free Rad Biol Med $1996 ; 20: 707$.

12. WITZTUM JL, STEINBERG D. The oxidative modification hypothesis of atherosclerosis: does it hold for humans? Trends Cardiovasc Med $2001 ; 11: 93$.

13. CHISOLM 3RD GM, CHAI Y. Regulation of cell growth by oxidized LDL. Free Radic Biol Med $2000 ; 28: 1697$

14. AUGE N, ESCARGUEIL-BLANC I, LAJOIE-MAZENC $\mathrm{I}$, et al. Potential role for ceramide in MAPK activation and proliferation of vascular smooth muscle cells induced by oxidized LDL. / Biol Chem $1998 ; 273: 12893$.

15. AUGE N, GARCIA V, MAUPAS-SCHWALM F, LEVADE T, SALVAYRE R, NEGRE-SALVAYRE A. Oxidized LDL-induced smooth muscle cell proliferation involves the EGF receptor/PI-3 kinase/Akt and the sphingolipid signaling pathways. Arterioscler Thromb Vasc Biol 2002; $22: 1990$. 
16. ESCARGUEIL-BLANC I, SALVAYRE R, VACARESSE $\mathrm{N}$, et al. Mildly oxidized LDL induce activation of PDGF ß-receptor pathway. Circulation 2001 ; $104: 1814$.

17. ROBBESYN F, SALVAYRE R, NEGRE-SALVAYRE A. Dual role of oxidized LDL on the NF-kappaB signaling pathway. Free Radic Res $2004 ; 38$ : 541.

18. MEILHAC O, ESCARGUEIL-BLANC I, THIERS JC, SALVAYRE R, NEGRE-SALVAYRE A. Bcl-2 alters the balance between apoptosis and necrosis, but does not prevent cell death induced by oxidized low density lipoproteins. FASEB $1999 ; 13: 485$.

19. ORRENIUS S, ZHIVOTOVSKY B, NICOTERA P. Regulation of cell death: the calcium-apoptosis link. Nat Rev Mol Cell Biol $2003 ; 4$ : 552.

20. PROSKURYAKOV SY, GABAI VL, KONOPLYANNIKOV AG. Necrosis is an active and controlled form of programmed cell death. Biochemistry (Mosc) $2002 ; 67: 387$.

21. LID, YANG B, MEHTA JL. Ox-LDL induces apoptosis in human coronary artery endothelial cells : role of PKC, PTK, bcl-2, and Fas. Am J Physiol 1998 ; 275(Pt 2) : H568.

22. SATA M, WALSH K. Oxidized LDL activates fasmediated endothelial cell apoptosis. I Clin Invest $1998 ; 102: 1682$.

23. WALTER DH, HAENDELER J, GALLE J, ZEIHER AM, DIMMELER S. Cyclosporine-A inhibits apoptosis of human endothelial cells by preventing release of cytochrome $\mathrm{C}$ from mitochondria. Circulation $1998 ; 98: 1153$.

24. HAJJAR DP, HABERLAND ME. Lipoprotein trafficking in vascular cells. Molecular Trojan horses and cellular saboteurs. / Biol Chem 1997 ; $272: 22975$

25. GAUT JP, HEINECKE JW. Mechanisms for oxidizing low-density lipoprotein. Insights from patterns of oxidation products in the artery wall and from mouse models of atherosclerosis. Trends Cardiovasc Med $2001 ; 11: 103$.

26. HEINECKE JW. Mechanisms of oxidative damage of low density lipoprotein in human atherosclerosis. Curr Opin Lipidol 1997 ; 8 : 268.

27. ESTERBAUER H, GEBICKI J, PUHL $H$, JÜRGENS $G$. The role of lipid peroxidation and antioxidants in oxidative modification of LDL. Free Radic Biol Med $1992 ; 13: 341$.

28. PARTHASARATHY S, SANTANAM N. Mechanism of oxidation, antioxidants, and atherosclerosis. Curr Opin Lipidol 1994 ; 5 : 371.

29. BJORKHEM I, DICZFALUSY U. Oxysterols: friends, foes, or just fellow passengers? Arterioscler Thromb Vasc Biol $2002 ; 22$ : 734.

30. COLLES SM, MAXSON JM, CARLSON SG CHISOLM GM. Oxidized LDL-induced injury and apoptosis in atherosclerosis. Potential roles for oxysterols. Trends Cardiovasc Med 2001 ; $11: 131$
31. BIASI F, LEONARDUZZI G, VIZIO B, et al. Oxysterol mixtures prevent proapoptotic effects of 7-ketocholesterol in macrophages: implications for proatherogenic gene modulation. FASEB / $2004 ; 18: 696$.

32. LEITINGER N. Oxidized phospholipids as modulators of inflammation in atherosclerosis. Curr Opin Lipidol $2003 ; 14: 421$.

33. PARTHASARATHY S, AUGE N, SANTANAM N Oxidized low-density lipoprotein, a two-faced Janus in coronary artery disease? Free Radic Res $1998 ; 28: 583$.

34. THOMAS SR, STOCKER R. Molecular action of vitamin $\mathrm{E}$ in lipoprotein oxidation: implications for atherosclerosis. Free Radic Biol Med 2000 ; $28: 1795$.

35. NEGRE-SALVAYRE A, VIEIRA O, ESCARGUEILBLANCI, SALVAYRER. Oxidized LDL and 4-hydroxynonenal modulate tyrosine kinase receptor activity. Mol Aspects Med 2003 ; 24 : 251.

36. HALLIWELL B, GUTTERIDGE JMC. Free radicals in Biology. Clarendon Press, 1989.

37. NILSSON I, DAHLGREN B, ARES M, et al. Lipoprotein-like phospholipid particles inhibit the smooth muscle cell cytotoxicity of lysophosphatidylcholine and platelet-activating factor. Arterioscler Thromb Vasc Biol $1998 ; 18$ : 13.

38. AUGE N, NEGRE-SALVAYRE A, SALVAYRE R, LEVADE T. Sphingomyelin metabolites in vascular cell signaling and atherogenesis. Prog Lipid Res $2000 ; 39$ : 207.

39. STEINBRECHER UP, GOMEZ-MUNOZ A, DURONIO V. Acid sphingomyelinase in macrophage apoptosis. Curr Opin Lipidol 2004 ; 15 : 531.

40. BROWN MS, GOLDSTEIN JL. Receptormediated endocytosis: insights from the lipoprotein receptor system. Proc Natl Acad Sci USA $1979 ; 76: 3330$.

41. KATAOKA H, KUME N, MIYAMOTO $S$, et al. Oxidized LDL modulates Bax/Bcl-2 through the lectinlike Ox-LDL receptor-1 in vascular smooth muscle cells. Arterioscler Thromb Vasc Biol $2001 ; 21: 955$.

42. WINTERGERST ES, JELK J, RAHNER C, ASMIS R. Apoptosis induced by oxidized low density lipoprotein in human monocyte-derived macrophages involves CD36 and activation of caspase-3. Eur J Biochem $2000 ; 267$ : 6050.

43. STEINBRECHER UP. Receptors for oxidized low density lipoprotein. Biochim Biophys Acta 1999 ; $1436: 279$.

44. FRANK PG, LISANTI MP. Caveolin-1 and caveolae in atherosclerosis: differential roles in fatty streak formation and neointimal hyperplasia. Curr Opin Lipidol 2004 ; 15 : 523.

45. CHEN K, KEANEY J. Reactive oxygen speciesmediated signal transduction in the endothelium. Endothelium $2004 ; 11$ : 109.
46. HSIEH CC, YEN MH, YEN CH, LAU YT. Oxidized low density lipoprotein induces apoptosis via generation of reactive oxygen species in vascular smooth muscle cells. Cardiovasc Res $2001 ; 49: 135$.

47. GALLE J, HEINLOTH A, WANNER C, HEERMEIER $K$. Dual effect of oxidized LDL on cell cycle in human endothelial cells through oxidative stress. Kidney Int $2001 ; 59$ : S120.

48. YELDANDI AV, RAO MS, REDDY JK. Hydrogen peroxide generation in peroxisome proliferatorinduced oncogenesis. Mutat Res 2000; 448 : 159.

49. COMINACINI L, RIGONIA, PASINIAF, et al. The binding of oxidized low density lipoprotein (ox-LDL) to ox-LDL receptor-1 reduces the intracellular concentration of nitric oxide in endothelial cells through an increased production of superoxide. / Biol Chem 2001; 279 : 13750.

50. LI W, YUAN XM, BRUNK UT. OXLDL-induced macrophage cytotoxicity is mediated by lysosomal rupture and modified by intralysosomal redox-active iron. Free Radic Res 1998; 29 : 389.

51. ROSENBLAT M, AVIRAM M. Macrophage glutathione content and glutathione peroxidase activity are inversely related to cell-mediated oxidation of LDL: in vitro and in vivo studies. Free Radic Biol Med 1998 ; 24 : 305.

52. SCHMITT A, NEGRE-SALVAYRE A, DELCHAMBRE J, SALVAYRE R. Prevention by alphatocopherol and rutin of glutathione and ATP depletion induced by oxidized LDL in cultured endothelial cells. Br / Pharmacol 1995 ; 116 : 1985.

53. SLOW RC, RICHARDS JP, REDLEY KC, LEAKEDS, MANN GE. Vitamin C protects human vascular smooth muscle cells against apoptosis induced by moderately oxidized LDL containing high levels of lipid hydroperoxides. Arterioscler Thromb Vasc Biol 1999; 19 : 2387.

54. VACARESSE $N$, VIEIRA O, ROBBESYN F, JURGENS G, SALVAYRE R, NEGRE-SALVAYRE A. Phenolic antioxidants trolox and caffeic acid modulate the oxidized LDL-induced EGFreceptor activation. Br / Pharmacol 2001 ; 132 : 1777.

55. ZHENG ZS, XUE GZ, GRUNBERGER D, PRYSTOWSKY JH. Caffeic acid phenethyl ester inhibits proliferation of human keratinocytes and interferes with the EGF regulation of ornithine decarboxylase. Oncol Res $1995 ; 7: 445$.

56. BRIGELIUS-FLOHE R, MAURER S, LOTZER K, et al. Overexpression of PHGPx inhibits hydroperoxide-induced oxidation, NFkappaB activation and apoptosis and affects oxLDLmediated proliferation of rabbit aortic smooth muscle cells. Atherosclerosis $2000 ; 152: 307$.

57. AUGE N, NEGRE-SALVAYRE A, SALVAYRE R, LEVADE T. Sphingomyelin metabolites in vascular cell signaling and atherogenesis. Prog Lipid Res $2000 ; 39$ : 207. 
58. AUGE N, NIKOLOVA-KARAKASHIAN M CARPENTIER $S$, et al. Role of sphingosine 1-phosphate in the mitogenesis induced by oxidized low density lipoprotein in smooth muscle cells via activation of sphingomyelinase, ceramidase, and sphingosine kinase. I Biol Chem $1999 ; 274: 21533$.

59. AUGE N, MAUPAS-SCHWALM F, ELBAZ M et al. Role for matrix metalloproteinase-2 in oxidized low-density lipoprotein-induced activation of the sphingomyelin/ceramide pathway and smooth muscle cell proliferation. Circulation $2004 ; 110$ : 571.

60. AUBIN I, ADAMS CP, OPSAHL $S$, et al. A deletion in the gene encoding sphingomyelin phosphodiesterase 3 (Smpd3) results in osteogenesis and dentinogenesis imperfecta in the mouse. Nat Genet 2005 ; $37: 803$.

61. STOFFEL W, JENKE B, BLOCK B, ZUMBANSEN $\mathrm{M}, \mathrm{KOEBKE}$ J. Neutral sphingomyelinase 2 (smpd3) in the control of postnatal growth and development. Proc Natl Acad Sci USA 2005 ; $102: 4554$.

62. STEINBRECHER UP, GOMEZ-MUNOZ A DURONIO V. Acid sphingomyelinase in macrophage apoptosis. Curr Opin Lipidol $2004 ; 15$ : 531.

63. DEIGNER HP, CLAUS R, BONATERRA GA, et al Ceramide induces aSMase expression : implications for oxLDL-induced apoptosis. FASEB $2001 ; 15: 807$.

64. ESCARGUEIL-BLANCI, ANDRIEU-ABADIE N, CASPAR-BAUGUIL $S$, et al. Apoptosis and activation of the sphingomyelin-ceramide pathway induced by oxidized low density lipoproteins are not causally related in ECV-304 endothelial cells. I Biol Chem 1998; 273: 27389.

65. SALVAYRE R, AUGE N, BENOIST H, NEGRESALVAYRE A. Oxidized LDL-induced apoptosis. Biochim Biophys Acta $2002 ; 1585$ : 213.

66. POLI G, LEONARDUZZI G, BIASI F, CHIARPOTTO E. Oxidative stress and cell signalling. Curr Med Chem $2004 ; 11$ : 1163.

67. VIEIRA O, ESCARGUEIL-BLANC I, JURGENS G, et al. Oxidized LDLs alter the activity of the ubiquitin-proteasome pathway : potential role in oxidized LDL-induced apoptosis. FASEB / $2000 ; 14: 532$.

68. BRAND K, BANKA CL, MACKMAN N TERKELTAUB RA, FAN ST, CURTISS LK, et al. Oxidized LDL enhances lipopolysaccharideinduced tissue factor expression in human adherent monocytes.Arterioscler Thromb 1994 ; $14: 790$.

69. BROWN AJ, MANDER EL, GELISSEN IC, KRITHARIDES L, DEAN RT, JESSUP W. Cholesterol and oxysterol metabolism and subcellular distribution in macrophage foam cells. Accumulation of oxidized esters in lysosomes. / Lipid Res $2000 ; 41: 226$
70. LIW, YUANXM, OLSSONAG, BRUNK UT. Uptake of oxidized LDL by macrophages results in partial lysosomal enzyme inactivation and relocation. Arterioscler Thromb Vasc Biol 1998 ; $18: 177$.

71. LI W, YUAN XM, BRUNK UT. Oxidized LDLinduced macrophage cytotoxicity is mediated by lysosomal rupture and modified by intralysosomal redox-active iron. Free Radic Res 1998 ; $29: 389$.

72. YUAN XM, LIW, BRUNK UT, DALEN H, CHANG YH, SEVANIAN A. Lysosomal destabilization during macrophage damage induced by cholesterol oxidation products. Free Radic Biol Med $2000 ; 28: 208$.

73. RIEDL SI, SHI Y. Molecular mechanisms of caspase regulation during apoptosis. Nat Rev Mol Cell Biol $2004 ; 11$ : 897

74. FERRI KF, KROEMER G. Organelle-specific initiation of cell death pathways. Nat Cell Biol $2001 ; 11$ : E255.

75. LEMASTERS JJ, QIAN T, HE L, et al. Role of mitochondrial inner membrane permeabilization in necrotic cell death, apoptosis, and autophagy. Antioxid Redox Signal $2002 ; 4$ : 769.

76. KOWALTOWSKIAJ, VERCESIAE, FISKUM G $\mathrm{BCl}-2$ prevents mitochondrial permeability transition and cytochrome $c$ release via maintenance of reduced pyridine nucleotides. Cell Death Differ $2000 ; 7: 903$

77. LEE T, CHAU L. Fas/Fas ligand-mediated death pathway is involved in oxidized LDL-induced apoptosis in vascular smooth muscle cells. Am J Physiol Cell Physiol 2001 ; 280 : C709.

78. ALCOUFFE J, THERVILLE N, SEGUI B, et al. Expression of membrane-bound and soluble FasL in Fas- and FADD-dependent T lymphocyte apoptosis induced by mildly oxidized LDL. FASEB / $2004 ; 18: 122$

79. WINTERGERST ES, JELK J, RAHNER C, ASMIS R. Apoptosis induced by oxidized low density lipoprotein in human monocyte-derived macrophages involves CD36 and activation of caspase-3. Eur J Biochem $2000 ; 267: 6050$.

80. DIMMELER S, HAENDELER J, GALLE J, ZEIHER AM. Oxidized low-density lipoprotein induces apoptosis of human endothelial cells by activation of CPP32-like proteases. A mechanistic clue to the 'response to injury' hypothesis. Circulation $1997 ; 95: 1760$.

81. VINDIS C, ELBAZ M, ESCARGUEIL-BLANC I, et al. Two distinct calcium-dependent mitochondrial pathways are involved in oxidized LDL-induced apoptosis. Arterioscler Thromb Vasc Biol $2005 ; 25$ : 639.

82. KAUFMAN RJ. Orchestrating the unfolded protein response in health and disease. / Clin Invest $2002 ; 110: 1389$.
83. RUTKOWSKI DT, KAUFMAN RJ. A trip to the ER : coping with stress. Trends Cell Biol 2004 ; $14: 20$.

84. FERRI KF, KROEMER G. Organelle-specific initiation of cell death pathways. Nat Cell Biol $2001 ; 3$ : E255.

85. SHIY, SAHAI BM, GREEN DR. Cyclosporin A inhibits activation-induced cell death in T-cell hybridomas and thymocytes. Nature 1989; $339: 625$.

86. CROAL DE, DEMARTINO GN. Calciumactivated neutral protease (calpain) system : structure, function and regulation. Physiol Rev $1991 ; 71: 813$

87. NEUMAR RW, XU YA, GADA H, GUTTMANN RP, SIMAN R. Cross-talk between calpain and caspase proteolytic systems during neuronal apoptosis. J Biol Chem 2003 ; 278 : 14162.

88. ESCARGUEIL-BLANC I, SALVAYRE R, NEGRESALVAYRE A. Necrosis and apoptosis induced by oxidized low density lipoproteins occur through two calcium-dependent pathways in lymphoblastoid cells. FASEB / 1994 ; 8 : 1075.

89. ESCARGUEIL-BLANC I, MEILHAC O, PIERAGGI MT, ARNAL JF, SALVAYRE R, NEGRESALVAYRE A. Oxidized LDLs induce massive apoptosis of cultured human endothelial cells through a calcium-dependent pathway. Prevention by aurintricarboxylic acid. Arterioscler Thromb Vasc Biol 1997 ; 17 : 331.

90. PORN-ARES MI, SAIDO TC, ANDERSSON T, ARES MP. Oxidized low-density lipoprotein induces calpain-dependent cell death and ubiquitination of caspase 3 in HMEC- 1 endothelial cells. Biochem / 2003 ; 374(Pt 2) : 403.

91. MIKITA T, PORTER G, LAWN RM, SHIFFMAN D. Oxidized low density lipoprotein exposure alters the transcriptional response of macrophages to inflammatory stimulus. / Biol Chem $2001 ; 276: 45729$.

92. SATA M, WALSH K. Endothelial cell apoptosis induced by oxidized LDL is associated with the down-regulation of the cellula caspase inhibitor FLIP. J Biol Chem 1998 ; 273 : 33103.

93. ASHKENAZI A, DIXIT VM. Apoptosis control by death and decoy receptors. Curr Opin Cell Biol $1999 ; 11: 255$

94. OHLSSON BG, ENGLUND MC, KARLSSON AL, et al. Oxidized low density lipoprotein inhibits lipopolysaccharide-induced binding of nuclear factor-kappaB to DNA and the subsequent expression of tumor necrosis factor-alpha and interleukin-1beta in macrophages. / Clin Invest $1996 ; 98: 78$. 
95. KATAOKA H, KUME N, MIYAMOTO $s$, et al. Oxidized LDL modulates $\mathrm{Bax} / \mathrm{Bcl}-2$ through the lectinlike Ox-LDL receptor-1 in vascular smooth muscle cells. Arterioscler Thromb Vasc Biol $2001 ; 21: 955$.

96. NAPOLI C, DE NIGRIS F, PALINSKI W. Multiple role of reactive oxygen species in the arterial wall. / Cell Biochem $2001 ; 82: 674$.
97. LEONARDUZZI G, ARKAN MC, BASAGA H, CHIARPOTTO E, SEVANIAN A, POLI G. Lipid oxidation products in cell signaling. Free Radic Biol Med $2000 ; 28: 1370$.

98. MAZIERE C, ALIMARDANI G, DANTIN F, DUBOIS F, CONTE MA, MAZIERE IC. Oxidized LDL activates STAT1 and STAT3 transcription factors : possible involvement of reactive oxygen species. FEBS Lett $1999 ; 448$ : 49.
99. HAMILTON TA, MAJOR JA, ARMSTRONG D, TEBO JM. Oxidized LDL modulates activation of NFkappaB in mononuclear phagocytes by altering the degradation if IkappaBs. I Leukoc Biol $1998 ; 64: 667$.

100. HEERMEIER K, LEICHT W, PALMETSHOFER A, ULLRICH M, WANNER C, GALLE J. Oxidized LDL suppresses NF-kappaB and overcomes protection from apoptosis in activated endothelial cells. J Am Soc Nephrol $2001 ; 12$ : 456. 